\title{
Nanomaterials in fluorescence-based biosensing
}

\author{
Wenwan Zhong
}

Received: 18 November 2008 /Revised: 29 December 2008 / Accepted: 21 January 2009 /Published online: 17 February 2009

(C) The Author(s) 2009. This article is published with open access at Springerlink.com

\begin{abstract}
Fluorescence-based detection is the most common method utilized in biosensing because of its high sensitivity, simplicity, and diversity. In the era of nanotechnology, nanomaterials are starting to replace traditional organic dyes as detection labels because they offer superior optical properties, such as brighter fluorescence, wider selections of excitation and emission wavelengths, higher photostability, etc. Their size- or shape-controllable optical characteristics also facilitate the selection of diverse probes for higher assay throughput. Furthermore, the nanostructure can provide a solid support for sensing assays with multiple probe molecules attached to each nanostructure, simplifying assay design and increasing the labeling ratio for higher sensitivity. The current review summarizes the applications of nanomaterials including quantum dots, metal nanoparticles, and silica nanoparticles - in biosensing using detection techniques such as fluorescence, fluorescence resonance energy transfer (FRET), fluorescence lifetime measurement, and multiphoton microscopy. The advantages nanomaterials bring to the field of biosensing are discussed. The review also points out the importance of analytical separations in the preparation of nanomaterials with fine optical and physical properties for biosensing. In conclusion, nanotechnology provides a great opportunity to analytical chemists to develop better sensing strategies, but also relies on modern analytical techniques to pave its way to practical applications.
\end{abstract}

Keywords Nanomaterials · Quantum dots · Gold nanoparticle $\cdot$ Silica nanoparticle $\cdot$ Fluorescence $\cdot$ FRET . Biosensing

W. Zhong $(\bowtie)$

Department of Chemistry, University of California,

Riverside, CA 92521, USA

e-mail: wenwan.zhong@ucr.edu

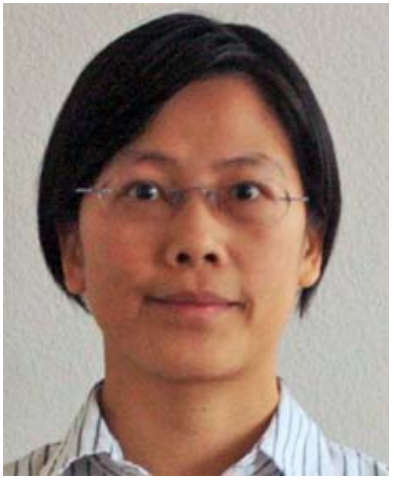

Wenwan Zhong

has been Assistant Professor of Chemistry at the University of California, Riverside, since July 2006. She received the Pilot Interdisciplinary Research Award from the Institute for Integrative Genome Biology of UC, Riverside. Her current research interests are: developing novel analytical strategies for utilizing nanomaterials in biosensing; studying nanotoxicity using microscale separation techniques like capillary electrophoresis; and developing field-flow fractionationbased methods for purification and analysis of large protein complexes.

\section{Overview}

Sensitive detection of target analytes present at trace levels in biological samples often requires the labeling of reporter molecules with fluorescent dyes, because fluorescence detection is by far the dominant detection method in the field of sensing technology, due to its simplicity, the convenience of transducing the optical signal, the availability of organic dyes with diverse spectral properties, and the rapid advances made in optical imaging. However, it can be difficult to obtain a low detection limit in fluorescence detection due to the limited extinction coefficients or quantum yields of organic dyes and the low dye-toreporter molecule labeling ratio. The recent explosion of nanotechnology, leading to the development of materials with submicron-sized dimensions and unique optical properties, has opened up new horizons for fluorescence detection. Nanomaterials can be made from both inorganic and organic materials and are less than $100 \mathrm{~nm}$ in length 
along at least one dimension. This small size scale leads to large surface areas and unique size-related optical properties. For example, the quantum confinement effects that occur in nanometer-sized semiconductors widen their band gap and generate well-defined energy levels at the band edges, causing a blueshift in the threshold absorption wavelength with decreasing particle size and inducing luminescence that is strictly correlated to particle size. Therefore, the position of the absorption as well as the luminescence peaks can be fine-tuned by controlling the particle size and the size distribution during synthesis, generating a large group of "fluorophores" with diverse optical properties. Another example is the collective oscillation of free electrons on the surfaces of noble metal particles when their sizes drop below the electron mean free path, which gives rise to intense absorption in the visible to near-UV region as well as a significant enhancement of the luminescence of the fluorophore nearby. On the other hand, they can be conjugated to the reporter molecules as optical tags, like organic dyes, due to their ultrafine physical sizes. Hence, this review focuses on the applications of nanomaterials, including semiconductor nanocrystals, noble metal nanoparticles, silica nanoparticles, and carbon nanotubes, in the field of fluorescence-based sensing.

\section{Fluorescent nanoparticle}

\section{Quantum dots}

Since their discovery, quantum dots (QDs) have become more and more important as fluorescence labels in biosensing and imaging [1-5]. They are semiconductor nanocrystals composed of atoms of elements from groups II to VI (e.g., Cd, Zn, Se, Te) or III-V (e.g., In, P, As) in the periodic table [3,5]. The quantum confinement effect arising from their very small $(<10 \mathrm{~nm})$ dimensions results in wide UV-visible absorption spectra, narrow emission bands, and optical properties that can be tuned by size, composition, and shape $[1,5]$. These features come with high flexibility in the selection of excitation wavelength as well as minimal overlap in the emission spectra from multiple QDs, making them excellent labels for highthroughput screening. Additionally, choosing excitation wavelengths far from the emission wavelengths can eliminate background scattering. Compared to organic dyes, QDs have similar quantum yields but extinction coefficients that are 10-50 times larger, and much-reduced photobleaching rates [2]. The overall effect is that QDs have 10 20 times brighter fluorescence and $\sim 100-200$ times better photostability [2].

Because QDs are intrinsically fluorescent, they can be employed as the reporter molecules for biomolecule detection. For example, QD-based western blot detection kits are able to detect as low as $20 \mathrm{pg}$ protein per lane [6, 7]. In comparison with colorimetric or chemiluminescent detection, which lead to detection limits of around hundreds of picograms of protein per lane, the QD-based protocol was found to require the same measuring time, be more sensitive, sustain a longer storage time after staining with minimal loss of signal, and deliver better image quality [11]. Innovative sensing formats have also been developed to utilize the special properties of QDs. A strategy for the smart targeting of protein was reported by Genin et al., which involved linking QDs to an organic dye of CrAsH [8]. Since the interaction between $\mathrm{CrAsH}$ and cysteine causes a significant increase in CrAsH's fluorescence, nanohybrids of CrAsH-QD could serve as a probe to locate Cys-tagged proteins and subsequently trace them for more than $150 \mathrm{~s}$, taking advantage of the high resistance of QDs to photobleaching [8]. On the other hand, Soman et al. profited from the much larger size of QDs than organic dyes when designing a protein detection scheme that offers subpicomolar sensitivity [9]. In this scheme, QD-Ab conjugates rapidly aggregate in the presence of antigens, resulting in colloidal structures that are 1-2 orders of magnitude larger in size than the constituents [9]. These structures are detected by light scattering in a flow cytometer [9]. The detection of various antigens using QDs with different emission properties is possible with this straightforward agglomeration-based detection strategy. Due to their bright intensity and high photostability, they also have a wide range of applications in bioimaging. For instance, QDs can be employed in fluorescence in situ hybridization (FISH) and offer higher detection sensitivity than organic dyes. QD FISH detected the expression of mRNA in neurons within the midbrain region of mouse at $\times 4$ magnification, which could only be done at $\times 20$ magnification when Alexa Fluor 488 was used [10]. Streptavidin-coated quantum dots bound to biotinylated peptides were produced in vivo after infection of the target bacterium by an engineered host-specific bacteriophage [11]. The system detected the specific bacterium at a concentration of ten bacterial cells per milliliter using flow cytometry or fluorescence microscopy [11]. The optical properties of QDs - a wide absorption spectrum, narrow emission peak, and high photostability-provide great advantages in high-throughput analysis [12-15]. Multiplexed detection of Bacillus anthracis was performed on a fiber-optic microarray platform using five types of QDs and the organic dyes $\mathrm{Cy} 3$ and Cy5. This eight-reporter system provides a fourfold throughput enhancement over standard two-color assays [14].

Even though QDs are a very promising replacement for traditional organic dyes in labeling biomolecules for bioassays and bioimaging, their surface properties need to 
be improved for better aqueous solubility and functionality, their stability should be enhanced, and their nonspecific binding to biomolecules needs to be decreased. Various methods have been developed for these purposes. Ligand exchange is a method commonly used to replace the hydrophobic capping molecules with bifunctional linker molecules for both enhanced solubility in water and to generate functional groups for chemical conjugation with biomolecules on the surface $[2,5]$. Another approach is to cover the hydrophobic surface groups through interactions with amphiphilic molecules like octylamine-modified polyacrylic acid [2,5]. This approach does not alter the surface and optical properties of QDs, because the original hydrophobic layer outside the core/shell CdSe/ZnS is intact. Polyethylene glycol (PEG) is another useful molecule for surface modification of QDs, because it is not only a good adapter for a variety of functional end-groups, such as biotin, amino, and carboxyl groups, but it can also help to improve the stability of QDs and decrease nonspecific binding [16]. It has been proven that QDs encapsulated in oligomerized PEG-phospholipid micelles are stable for weeks in pure water (no change in precipitation and fluorescence was observed), and over $90 \%$ of the fluorescence was retained after 5,000 min when the oligomeric PEG-phospholipid micelle QDs were dispersed in acetate, phosphate, and borate buffers with low salt contents. In contrast, the fluorescence of monomeric PEG-phospholipid micelle QDs dropped drastically to $<50 \%$ or even $30 \%$ under the same conditions [17]. Surface oxidation and $\mathrm{pH}$ value during storage are two prominent factors that influence QD stability. For extended stability in basic buffers, dihydrolipoic acid (DHLA) could be attached to the QD surface via a PEG linkage [18]. The hydroxylcoated QDs prepared by Kairdolf et al. showed stability under both basic and acidic conditions, with minimized nonspecific binding [19]. All of these surface modification routes strengthen the compatibility of QDs with bioassays and should be continued in order to further enhance their applicability in the field of biological science.

Toxicity is another issue that needs to be solved before QDs can be widely applied in biomedical studies performed in vivo, although it may not be a big problem in biosensing performed in vitro.

\section{Fluorescent silica nanoparticles}

Another type of fluorescent nanomaterial which has been extensively tested as a labeling reagent in the detection of pathogens, nucleic acids, and proteins is silica nanoparticles doped with organic dyes [20-25]. This type of nanomaterial has the following advantages in biosensing: (1) silicon is abundant and nontoxic; (2) the high surface-to-volume ratio of the nanoparticles facilitates their binding to biomole- cules; (3) the inclusion of a large number of fluorescent dye molecules inside each nanoparticle results in excellent photostability due to the ability of the silica matrix to shield from molecular oxygen, and the inclusion also dramatically increases the dye-to-biomolecule labeling ratio, leading to high signal amplification factors during detection; and (4) silica is relatively inert in chemical reactions, but still allows surface modification with wellestablished chemistries [20,21]. Compared to QDs, fluorescent silica nanoparticles have a wider size range, spanning from a few to hundreds of nanometers; they require less strict size control, and exhibit better water solubility $[20,21]$. However, problems related to particle aggregation and nonspecific binding on the silica surface have been observed and will need to be solved before the full potential of silica nanoparticles in biosensing can be realized [20]. Studies have shown that a ratio of inert to active functional groups on the surfaces of silica nanoparticles that results in a high zeta potential is critical to maintaining a well-dispersed nanoparticle suspension and reducing nonspecific binding [26].

Other than organic fluorophores, silica particles can also encapsulate quantum dots. The encapsulation not only retains the unique optical properties of QDs, but it also eliminates the aqueous solubility and modification problems associated with QDs and reduces the toxicity of QDs by preventing the leakage of heavy metal ions into the environment [27]. Furthermore, magnetic nanoparticles can be co-embedded with QDs to allow handy manipulation of the particles using a magnetic field. Such multifunctional particles could be employed in live cell imaging [27-30].

Since toxicity is always a big concern when using cadmium-containing QDs in biomedical research, efforts have been expended to generate silicon QDs (SiQDs) that are far less toxic than group II-VI QDs. However, the indirect bandgap character of silicon results in the extremely low light emission efficiency. Recently, silicon quantum dots with photoluminescence quantum yields of over $60 \%$ have been demonstrated for organically capped silicon nanocrystals, with emission in the near-infrared range [31, 32]. Other big challenges in making biocompatible SiQDs include the instability of their photoluminescence due to their fast oxidation rate in aqueous environments, and the difficulties involved in attaching hydrophilic molecules to the SiQD surface [33-36]. Optimal surface functionalization, such as capping the surface of the SiQD with $\mathrm{NH}_{2}$, $\mathrm{SH}, \mathrm{OH}$, acrylic acid, and alkyl groups, has been sought to produce water-dispersible SiQDs while maintaining spectral and colloidal stability [33-36]. Highly stable aqueous suspensions of SiQDs encapsulated in phospholipid micelles were prepared and applied as luminescent labels for pancreatic cancer cells [35]. However, applications of 
SiQDs in biosensing and biomedical imaging are still in their infancy because the mechanism responsible for the visible photoluminescence (PL) of SiQDs and the relationship of PL to surface functionalization are not yet clear [34]. Moreover, a comprehensive comparison of the optical properties of organic dyes or Cd-based QDs with SiQDs has not been made, which makes it impossible to assess the analytical performance of SiQDs in biosensing.

\section{Metallic nanomaterials for fluorescence enhancement}

It has been known for a long time that metallic nanostructures can enhance the fluorescence of nearby fluorophores [37-39]. Interactions between the dipole moments of the fluorophores and the surface plasmon field of the metal can increase the incident light field, which results in enhanced local fluorescence intensity and rate of excitation. Such interactions also boost the radiative decay rates, leading to an improved quantum yield and a reduced lifetime of the fluorophore [37-39]. It has been estimated that the local electric field and the radiative rate could be increased by factors of 140 and 1,000, respectively, near a silver particle [38]. In addition, the shorter lifetime comes with the advantage of higher photon flux and increased photostability [37, 38]. The combined effect is the amplification of the total number of detected photons per fluorophore by a factor of $10^{5}$, significantly enhancing the detectability of the fluorophore [38].

Surfaces on which silver islands or silver particles have been deposited are common platforms in bioassays utilizing $\mathrm{MEF}$ as the reporting system. Enhancements of the detection signal ranging from ten- to fortyfold have been observed on silver island film ( $\mathrm{SiF}$ )-coated glass surfaces in comparison with the bare glass [40, 41]. An approximately thirtyfold increase in the fluorescent intensity of indocyanine green was observed on a silver colloid-coated planar surface [42]. The distance from the fluorophore to the metal surface is a critical factor in successful fluorescence amplification, because a distance that is too short can lead to quenching of the dye [43]. Such a distance dependence of the transfer of electronic energy from a donor plane of molecules to an acceptor plane was modeled by Aroca et al. in the early 1990s [44]. The ideal range is 50-200 $\AA$ from the metal surfaces, which makes conjugation of dye-labeled molecules on the silver surface necessary for MEF [38, 43]. The thickness of the silver coating also plays an important role in MEF. A study conducted by Zhang et al. found that the fluorophore was quenched on a silver film of $2 \mathrm{~nm}$, enhanced on a film thicker than $5 \mathrm{~nm}$, and reached saturation at $20 \mathrm{~nm}$ [45]. Anisotropic silver nanostructures have been constructed for MEF as well because theoretical calculations indicated that the surface of a spheroid with an aspect ratio of 1.75 could lead to a greater reduction in the radiative decay rate than that of a sphere or a more elongated spheroid [43, 46]. A fiftyfold fluorescence enhancement was observed with high loading of nanorods on the surface, and triangular silver plates led to an enhancement factor of 16 [46].

Metals other than silver have been tested for their effects on MEF. For instance, the use of a gold nanostructure coupled to $\mathrm{CdSe} / \mathrm{ZnS}$ nanocrystals for MEF was demonstrated with precise control and high spatial selectivity over the fluorescence enhancement process [47]. While silver or gold nanostructures achieve MEF in the visible to nearinfrared region, aluminum nanoparticles deposited on $\mathrm{SiO}_{2}$ substrate could work in the ultraviolet-blue spectral region, which broadens the application range of MEF [48]. Copper particulate films were also found to generate a moderate enhancement effect [49].

MEF has been employed to increase the sensitivity in the detection of DNA, RNA, and proteins in the microarray format [50-52]. A twenty-eight-fold fluorescence enhancement was observed for the near-infrared/infrared dye Cy5, but only a fourfold enhancement was obtained with $\mathrm{Cy} 3$, probably due to the stronger scattering component of the extinction spectrum of Cy5 (Fig. 1) [52]. Sensitive detection of a 484-mer RNA with a detection limit of 25 fmol (4 ng) has been demonstrated with MEF and a detection probe consisting of a TAMRA-labeled DNA oligo [53]. Detections of a few nanograms of RNA in RNA capture assays had only been achieved previously with enzymatic signal amplification via alkaline phosphatase or linear mRNA amplification during cDNA synthesis, which are more complicated and time-consuming processes than the MEF-based method [54, 55]. Proof-of-principle detection of proteins has been demonstrated using model systems like avidin-biotin and antigen (myoglobin)-antibody [51]. Moreover, silver island film and MEF have the potential to increase the sensitivities of bioassays performed on cell membranes. Cells were simply cast onto glass slides covered with silver islands and dried before measurement [56]. Because the fluorescence signals of the fluorophores bound on the cell membranes were enhanced dramatically by the $\mathrm{SiF}$ supports while the intensity level of the intracellular fluorophores was not changed, the MEF helped to distinguish membrane-bound signals from those inside the cells [56]. In order to spatially and kinetically accelerate the binding of biomolecules onto the surface, microwave heating was employed to enable ultrafast detection by MEF $[57,58]$. The microwave-based approach also facilitated the release of genomic materials from bacteria spores. Extraction and detection of DNA materials from less than 1000 Bacillus anthracis spores have been achieved within one minute by the microwave-assisted MEF technique [59]. Without MEF, Taqman $^{\circledR}$ real-time PCR was needed to 


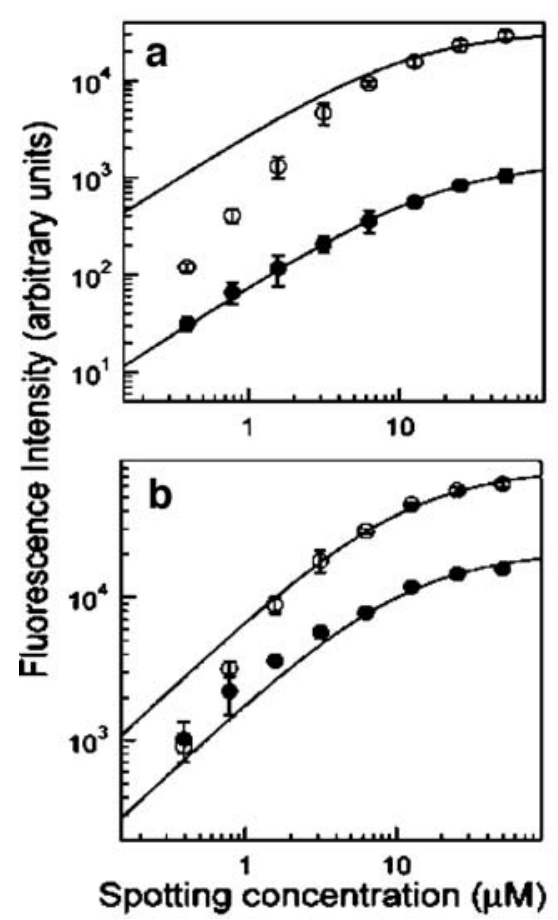

Fig. 1 Comparison of the fluorescence on glass and on SiF. A Cy5 fluorescence on glass (filled circles) and SIF (empty circles). B Cy3 fluorescence on glass (filled circles) and SIF (empty circles). C Plots of the intensity enhancement factor versus spotting concentration. Cy5

detect the same DNA materials from 100 unprocessed bacterial spores within $2 \mathrm{~h} \mathrm{[60].}$

The use of biomolecule-conjugated silver nanoparticles allows MEF to be applied to solution-based assays as well [61]. Solution-based sensing offers faster reaction kinetics and requires simpler experimental apparatus. Silica-coated silver spheres conjugated with $\mathrm{Cy} 3$ through streptavidinbiotin binding resulted in three- to five-fold fluorescence enhancements [61]. Fluorescent core-shell $\mathrm{Ag} @ \mathrm{SiO}_{2}$ nanoballs were also synthesized by the same group. The silver nanoball was coated with the fluorophore-doped silica shell, and a twentyfold increase in fluorescence was observed with this structure compared to nanobubbles without a silver core (Fig. 2) [62].

\section{Fluorescence resonance energy transfer (FRET)}

For assays studying biomolecule interactions and conformational changes, FRET is a better technique than simple fluorescence because it is very sensitive to nanoscale changes in distance between molecules [63]. Traditional FRET pairs are organic dyes, and modern nanotechnology produces materials like single metal nanoparticles and ionic nanocrystals that can be used as FRET donors or acceptors,
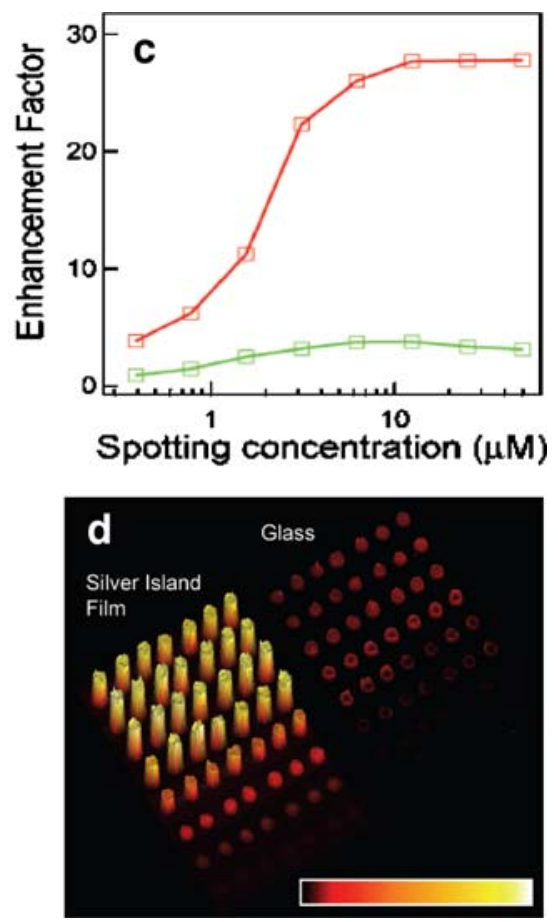

is shown in red, and Cy3 is shown in green. D A Cy5 scan for cohybridization with complementary $\mathrm{Cy} 5$ - and $\mathrm{Cy} 3$-labeled targets on glass and SIF substrates. The intensity bar shown lower right is a linear scale from 0 counts (black) to 34,000 counts (white) [52]

offering better FRET effects and more flexible sensing platforms in bioanalysis [63].

\section{Metal nanoparticles (NP)}

Gold nanoparticles are excellent FRET-based quenchers because their plasmon resonance in the visible range makes them strong absorbers and scatterers, with large extinction coefficients of around $10^{5} \mathrm{~cm}^{-1} \mathrm{M}^{-1}$. Additional superior optical properties include stable signal intensities and photobleaching resistance [63]. Gold NP is exceptionally attractive in bioassays due to the ability to finely control particle sizes, and the extreme ease with which biomolecules containing exposed thiol groups can be attached to the gold surface through gold-sulfur bonds. The gold-sulfur bond also facilitates the attachment of other functional groups such as carboxyl and amine groups via sulfurcontaining ligands with special terminal groups. The most typical application of gold NP in FRET-based assays is the labeling of molecular beacons [63]. The ends of the hairpin structure are conjugated to Au NP and organic fluorophore, respectively. When the molecular beacon opens up its stem upon binding to its complementary strand in the loop, the fluorophore is released from the $\mathrm{Au} \mathrm{NP}$ and increased fluorescence intensity is observed. A hundredfold increase 

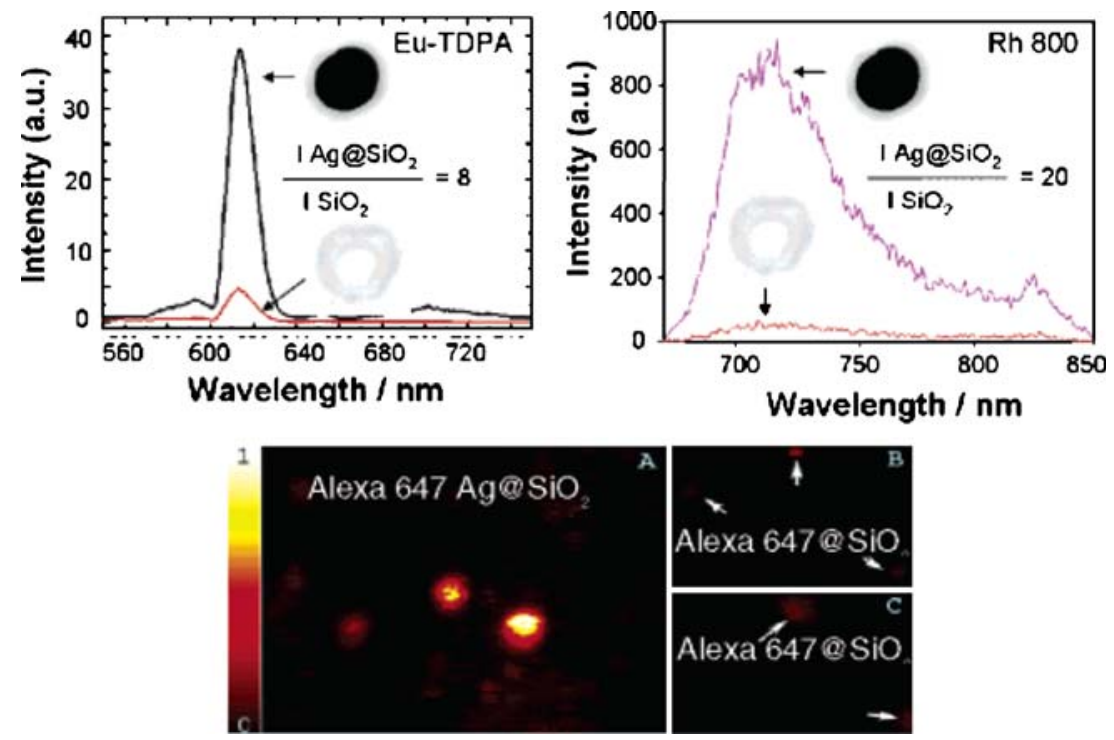

Fig. 2 Fluorescence emission intensities of Eu-TDPA-doped $\mathrm{Ag} @ \mathrm{SiO}_{2}$ and $\mathrm{Rh} 800$-doped $\mathrm{Ag} @ \mathrm{SiO}_{2}$, as well as those of the corresponding fluorescent nanobubbles, Eu-TDPA-doped $\mathrm{SiO}_{2}$ and $\mathrm{Rh} 800$-doped $\mathrm{SiO}_{2}$ (top). The bottom of the figure presents scanning confocal images of $(A)$ Alexa $647 \mathrm{Ag} @ \mathrm{SiO}_{2},(B)$ Alexa 647@ $\mathrm{SiO}_{2}$, and $(C)$ zoomed-in version of panel $(B)$. TDPA, tris(dibenzoylmethane) mono(5-aminophenanthroline) europium; $R h$, rhodamine [62] in sensitivity was obtained with Au NP compared with traditional dye combinations $[64,65]$. Bridge molecules can also be employed to bring the fluorophores into the proximity of Au NP. For example, $\beta$-cyclodextrin (CD) attached to the $\mathrm{Au}$ NP surface formed inclusion complexes with the fluorescein molecules which was then quenched [66]. This can be utilized to detect cholesterol, because the replacement of fluorescein at the binding site by cholesterol frees the fluorescein from the NP. The fluorescence intensity of the system increased in proportion to the cholesterol concentration [66]. The small particle size of Au NP makes them excellent in vivo imaging reagents as well. Au NP modified with FAM-labeled single-stranded DNA was used to image intracellular hydroxyl radicals; here, the DNA strand was cleaved by $\mathrm{HO}$. and released the quenched fluorophore [67].

Silver nanoparticles can be excellent acceptors in FRET too, or they can act as enhancers for FRET pairs. It has been found that hybridization of the donor-labeled oligonucleotide with the acceptor-labeled complimentary strand on a silver particle surface led to enhanced FRET efficiency with increased Förster distance (from 8.3 to $13 \mathrm{~nm}$ ) and a 21-fold faster FRET rate constant [68]. Furthermore, the efficiency of FRET between Cy5 and Cy5.5 on the surfaces of silver particles was increased when the particle size was increased from $15 \mathrm{~nm}$ to $80 \mathrm{~nm}$ and when the distance of the donor-acceptor pair from the particle surface was increased from $2 \mathrm{~nm}$ to $10 \mathrm{~nm}$ [69]. Therefore, as in the case of MEF, silver particles or a silver-decorated surface can act as the supporting material for FRET-based sensing to enhance assay performance.

\section{Quantum dots}

Other than being directly used as fluorescent labels, QDs have also been widely recognized recently as energy donors in FRET for bioanalysis. Their broad absorption and narrow emission spectra allow single-wavelength excitation of multiple donors and can avoid crosstalk with acceptor fluorophores. In addition, the spectral overlap between donors and acceptors can be adjusted by tuning the particle size. Moreover, QDs are nanostructures that can be coupled to multiple acceptor fluorophores for higher efficiency in energy transfer and can act as the solid support for biomolecules for imaging purposes or to simplify assay design. Last but not least, the energy transfer between QDs and molecular dyes can be approximately described by the Förster mechanism, and so accurate measurements of donor-acceptor distances can be deduced using the same FRET theory as for organic dyes when QDs are the donors and the organic dyes or QDs are the acceptors [70]. However, because of the broad absorption spectra and the long excitation lifetime of QDs, they are not suitable being energy acceptors for short-lived molecular fluorophores [71]. FRET-based analyses of nucleic acids, proteins, and other biological molecules have been reviewed extensively by Algar et al. Applications of QDs as the donors in FRET for DNA point mutation analysis, detection of pathogenic DNA, construction of molecular beacons with increased photostability, and immunoassays were covered in that review and will not be discussed here [71]. Some new applications of QDs as FRET probes included detection of 
Fig. 3 A Conceptual scheme for a single-QD-based nanosensor for evaluating Rev peptideRRE interactions and the inhibitory efficacy of proflavin based on FRET between 605QD and Cy5. B Histograms of the measured FRET efficiency for 605QD/RRE-Rev peptide/Cy5 complexes as a function of increasing Rev peptide-to-RRE ratio. The solid curves represent the fit of the experimental data to a Gaussian function. $\mathbf{C}$ The variation in the number of $\mathrm{Cy} 5$ burst counts with increasing Rev peptide-to-RRE ratio [74]

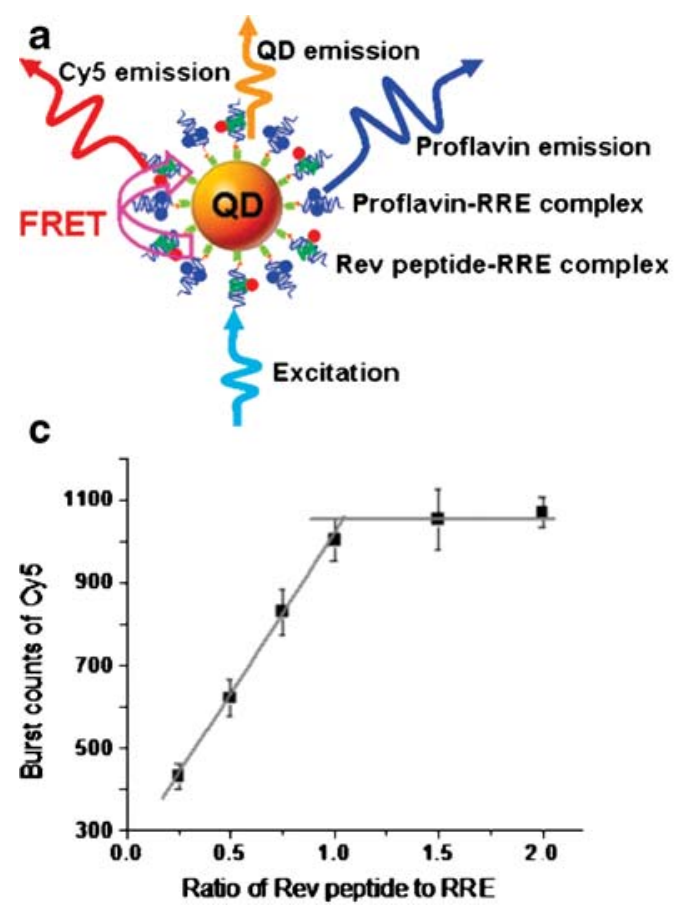

the actions of biological enzymes such as protease, polymerase, and nuclease, or even visualization of the $\mathrm{pH}$ change in solution, as demonstrated by Suzuki et al. [72]. Multiplexed detection of trypsin and deoxyribonuclease was also demonstrated [72]. On the other hand, QDmediated FRET can contribute greatly to the process of drug discovery and development. For example, it can be employed to image the cargo-unpacking process that occurs inside cells during drug delivery. The plasmid DNA is labeled by QDs and the polymeric gene carriers, such as chitosan and polyethyleneimine, are labeled with Cy5 [73]. The dissociation of plasmid DNA from the polymeric carriers after entering the cells releases the fluorescence of Cy5, as visualized by confocal microscopy [73]. In another b

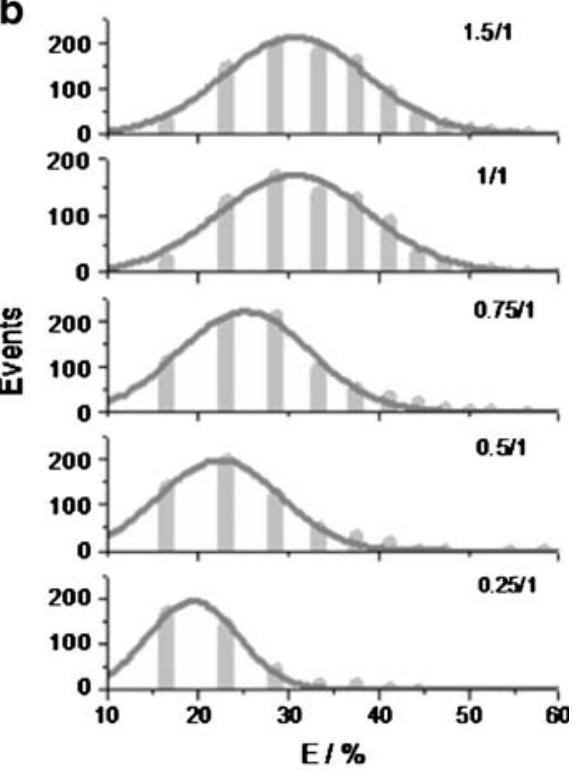

Fig. 4 Chemical structure of the QD-Con A- $\beta$-CD-Au NP nanobiosensor, and schematic illustration of its FRET-based operating principles [76]

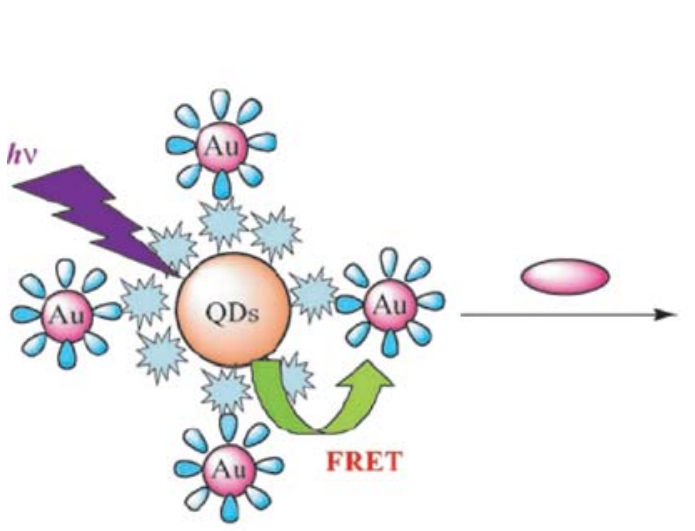

FRET effect on

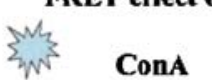

example, QD-based FRET was employed to quantify RNA-peptide interactions that could be applied in the screening of libraries of small-molecule drugs (Fig. 3) [74]. The important HIV-1 regulatory protein Rev was labeled with Cy5, and the Rev responsive element on HIV RNA (RRE RNA) was bound to the 605QD via the biotinstrapavidin interaction [74]. Association of Rev with the RRE RNA permitted the excitation of Cy5 at $444 \mathrm{~nm}$, a wavelength outside of the excitation range of $\mathrm{Cy} 5$, which would then decrease upon the binding of a small-molecule inhibitor like proflavin [74]. Using the 605QD as the FRET donor not only eliminated the interference from the intrinsic fluorescence of the inhibitor (proflavin), the emission spectrum of which significantly overlaps with the absorp- 
tion spectrum of Cy5, but it also provided a nanoscaffold that locally concentrated multiple copies of Cy5-labeled Rev on the QD surface, achieving signal amplification. Similar designs would be highly useful in high-throughput drug screening.

Another unique FRET system based on nanomaterials is the gold NP (donor)-QD (acceptor) pair. The combination of the large extinction coefficient of gold NPs and the bright fluorescence of QDs offers improved sensitivity and lower background in FRET measurement [75]. Successful applications of the Au-QD assembly were demonstrated with either simple systems like DNA hybridization and biotin-avidin binding or by detecting biologically significant substances in complex matrixes [63]. Taking advantage of the competition between $\beta-\mathrm{CD}$ and glucose in binding to the protein concanavalin A (Con A), a nanosensor of CdTe QD-Con A- $\beta-C D-A u$ NP quantified glucose in adult human serum with a detection limit as low as $50 \mathrm{nM}$ and excellent selectivity over other sugars and coexisting biological species (Fig. 4) [76].

\section{Silica nanoparticles}

There have been two approaches to utilizing silica nanoparticles in FRET-based sensing. One is to use them as the solid support for the occurrence of FRET to facilitate material handling. This approach has been applied to detect TNT by utilizing the specific binding between TNT and amines (Fig. 5A) [77]. A strong charge transfer interaction between the electron-deficient aromatic ring of TNT and the electron-rich amino group coupled to the silica particle surface resulted in strong absorption at $520 \mathrm{~nm}$ and relatively weak absorption at $630 \mathrm{~nm}$ (Fig. 5B) [77]. Therefore, the TNT-amine complex could act as the energy acceptor for FITC or ROX conjugated in the proximity of the amine groups [77]. The silica particles could be deposited into the microwells on a silicon wafer to form a convenient device for detecting trace TNT in solutions or air vapor (Fig. 5C) [77]. Similarly, a label-free DNA detection apparatus was constructed by conjugating the capture DNA probe onto the surfaces of silica particles [78]. Hybridization of the target DNA allowed intercalation of ethidium bromide (EB) - the FRET acceptor, and electrostatic binding of tetrahedralfluorene-the FRET donor [78]. Distinguishable fluorescence signals from EB were observed from the perfect match strand and from strands with single- or two-base mutations [78].

The other approach is to dope multiple energy transfer fluorescent dyes into silica NPs. By varying the doping ratios of the three tandem dyes, 5, (6) carboxylfluorescein (FITC), 5, (6) carboxylrhodamine 6G (R6G), and 6carboxy-X-rhodamine (ROX), FRET-mediated emission
Fig. 5 A Schematic illustration of FRET-based silica nanoparticle sensors for TNT detection. B The absorption and emission spectra of (1) FITC-( $\left.\mathrm{NH}_{2}\right)$-silica and (2) ROX-( $\left.\mathrm{NH}_{2}\right)$-silica nanoparticle solutions. The inset shows optical images of (1) FITC-( $\left.\mathrm{NH}_{2}\right)$-silica and (2) ROX-( $\left(\mathrm{NH}_{2}\right)$-silica nanoparticle solutions under natural light (left) and under a 360-nm UV lamp (right), respectively. C Regular array assembly of FITC-( $\left.\mathrm{NH}_{2}\right)$-silica nanoparticles on the silicon wafer with etched microwells. Confocal fluorescence images show the evolution in the brightness and size of the fluorescent dots upon adding $10 \mu \mathrm{L}$ of TNT solution of different concentrations [77]

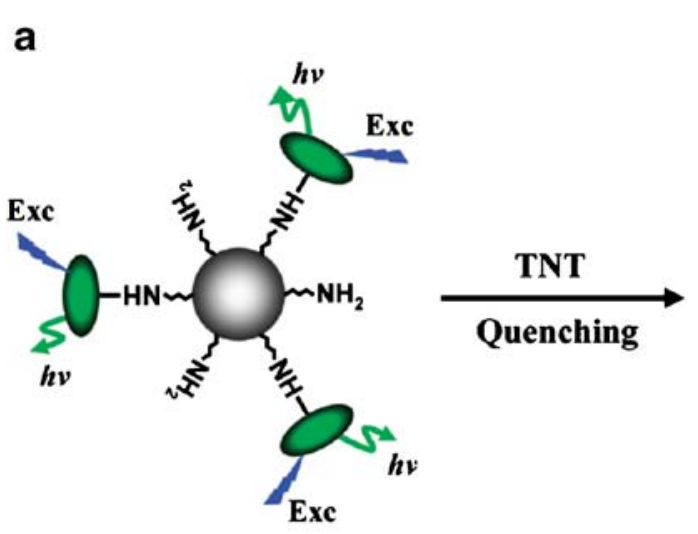

b

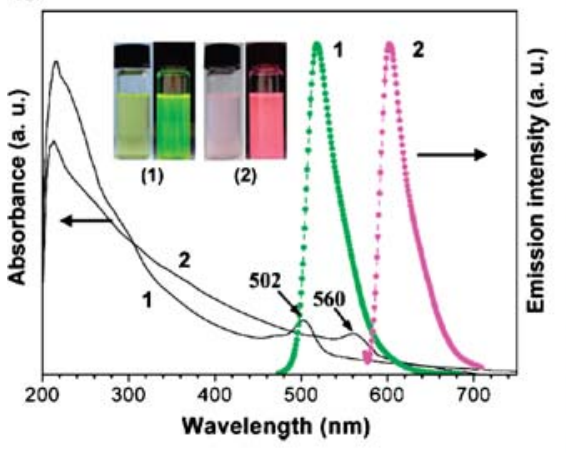

C
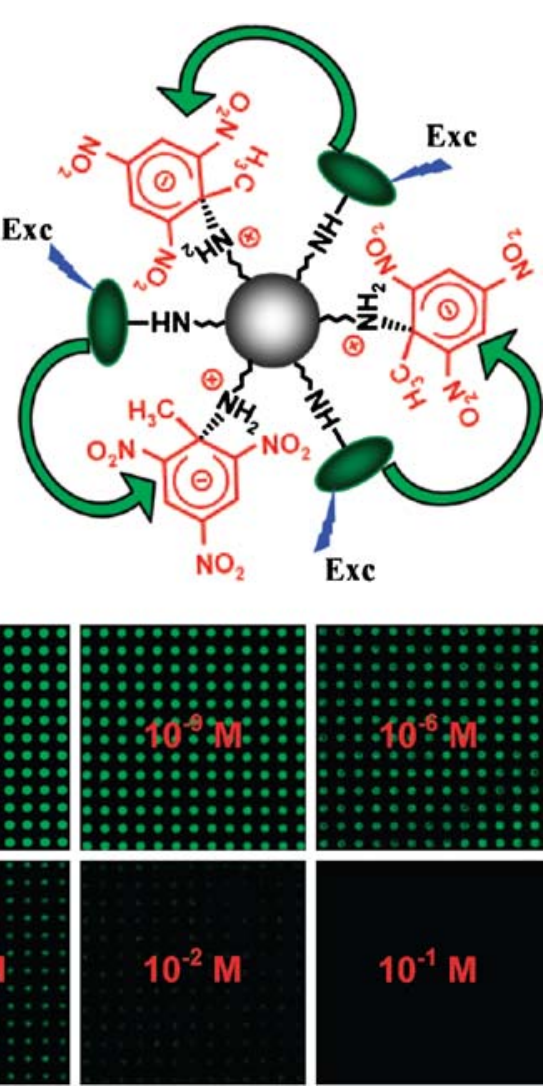
signatures can be identified, and the silica particles can emit multiple colors at a single excitation wavelength [79]. Such particles were applied to specifically detect three pathogenic bacteria species (Escherichia coli, Salmonella typhimurium, and Staphylococcus aureus) in solution [80].

\section{Alternative strategies for fluorescence-based sensing with nanomaterials}

Quenching with carbon nanotubes

Carbon nanotubes (CNTs) represent another type of unique nanomaterial used in fluorescence-based bioassays. The sensing utilizes the ability of CNTs to quench organic dyes or QDs [81-83]. An investigation of the effects of multiwall CNTs on the photoluminescence properties of CdSe QDs showed that CNTs could suppress the photoluminescence (PL) of QDs through both dynamic and energy transfer quenching mechanisms [83]. In order to potentially exploit this feature in bioassays, self-assembly of the oligonucleotide-modified QDs and CNTs through hybridization was studied, and sensitive detections of DNA and antigen down to the $0.2 \mathrm{pM}$ and $0.01 \mathrm{nM}$ levels, respectively, were achieved [81]. Similarly, organic dyes could be quenched by CNTs through an energy transfer mechanism [84-86]. This feature was employed to develop a noncovalent assembly between CNT and ssDNA for effective sensing of biomolecule interactions (Fig. 6) [82]. The strong interaction between CNT and ssDNA quenched the fluorophore conjugated on ssDNA. Hybridization of a complimentary DNA strand or binding of an interactive protein caused ssDNA to be released from the CNT and restored the fluorescence signal [82].

Fluorescent signal amplification with nanomaterials

Instead of generating highly fluorescent nanoparticles, different strategies have been developed to amplify the fluorescent signal in biosensing using nanomaterials. One of them is to increase the fluorescent dye-to-biomolecule labeling ratio by nanocarriers, so that each label can lead to fluorescence from hundreds or thousands of fluorophores. The FloDots we mentioned before utilize this strategy, encapsulating many fluorophores within each silica nanoparticle. Materials other than silica, such as polystyrene and liposomes, can also be used, but the relatively large size of the latex microspheres and the chemical instability of liposomes limit their applications in modern biosensing. Recently, fluorescent dye-doped conjugated polymer nanoparticles with an average size of $30 \mathrm{~nm}$ have been synthesized which offer several features, including high fluorescence intensity, a highly redshifted emission spectrum, and outstanding photostability [87]. The conjugated polyfluorene is one of this type, which possesses excellent harvesting ability for encapsulated fluorophores of perylene or coumarin 6 [87]. The polymer acted as a highly efficient energy donor to the dye molecule and the high efficiency of the energy transfer was attributed to the combined effects of energy diffusion, Föster transfer, and particle size [87]. The authors demonstrated that such particles had an approximately 200-fold higher fluorescence than QDs and were
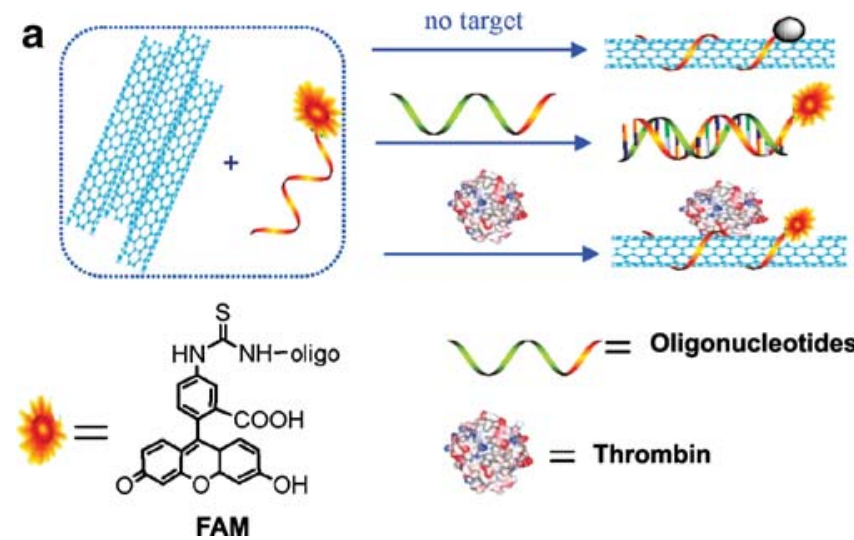

Fig. 6 A Scheme for signaling biomolecular interactions using an assembly between SWNTs and dye-labeled ssDNA. $P 1$ and $P 2$, the FAM-labeled oligonucleotides; $P 2$, the thrombin-binding aptamer; $T 1$ and T2, the perfect cDNA (T1) and one mismatched DNA (T2) of P1. B Fluorescence emission spectra $\left(\lambda_{\mathrm{ex}}=480 \mathrm{~nm}\right)$ of P1 $(50 \mathrm{nM})$ under

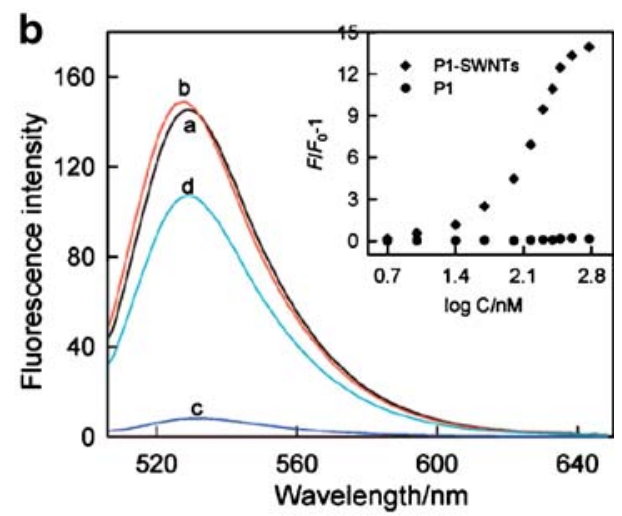

different conditions: (a) P1 in PBS; (b) P1 + $300 \mathrm{nM} \mathrm{T1}$; $(c) \mathrm{P} 1+$ SWNTs; and $(d) \mathrm{P} 1+\mathrm{SWNTs}+300 \mathrm{nM}$ T1. Inset: fluorescence intensity ratio of $\mathrm{P} 1(b)$ and $\mathrm{P} 1-\mathrm{SWNT}$ with $F / F_{0}-1$ plotted against the logarithm of the concentration of T1 [82] 
40 times brighter than silica nanoparticles of a comparable size that had been doped with the same dye molecules [87]. Thus they could be promising labeling agents in biosensing.

On the other hand, nonfluorescent materials can be loaded into nanoparticles and then released as fluorescent molecules for signal amplification. A low fluorescence background, controlled release, and reduced dye quenching when the loaded dye concentration is increased are attractive features of such materials. Hollow periodic mesoporous organosilica (PMO) spheres with multiple polyelectrolyte coatings for bioconjugation were prepared in order to encapsulate nonfluorescent fluorescein diacetate (FDA) [88]. The silica spheres could be dissolved in $\mathrm{NaOH}$, which also hydrolyzes the FDA to generate the fluorescent molecule fluorescein (Fig. 7) [88]. A high fluorescent dye-to-biomolecule labeling ratio of 1,000 6,000 was achieved in order to generate a fluorescent signal that was about 50 times higher than that of the conventional label [88]. However, the spheres had a relatively large size of $400 \mathrm{~nm}$. A large label usually generates problems related to the reduced activity of conjugated molecules and spatial hindrance on the sensing platform.

Recently, a novel fluorescence amplification strategy was developed by our group [89]. It was discovered that cation exchange $(\mathrm{CX})$ reactions could occur completely and reversibly in ionic nanocrystals at room temperature with unusually fast reaction rates because of their large surface area and small volume [90]. Our design takes advantage of this special feature to release the cations from the nanocrystals by cation exchange in ionic nanocrystals, which in turn trigger fluorescence in metal-responsive fluorophores [89]. Therefore, a large enhancement of the fluorescent signal amplification is achieved. A proof-of-principle study employing the nanocrystal-dye set of the nonfluorescent CdSe and Fluo-4, and using $\mathrm{Ag}^{+}$to exchange $\mathrm{Cd}^{2+}$ out of $\mathrm{CdSe}$, led to a sixtyfold higher fluorescent signal and a hundredfold lower detection limit in protein detection compared to the organic fluorophore Alexa Fluor 488 [89]. This signal amplification scheme is simple and fast, with a large dye-to-reporter labeling ratio, and has no issues with quenching. Our study also indicated that other nanocrystals such as $\mathrm{PbS}$ and metal-sensitive fluorophores like Rhod- $5 \mathrm{~N}$ could be chosen to further improve detection performance. In contrast to other detection schemes that utilize high-quality nanomaterials with special optical properties, our approach employs nonfluorescent nanocrystals that could be available at a much reduced cost.

\section{Nanomaterials in other fluorescence-based measurements}

Nanomaterials provide benefits over other fluorescencebased measurement techniques like fluorescence lifetime measurement and multiphoton fluorescence microscopy.
Fig. 7 A The transfer of fluorescein diacetate to the dianionic form of fluorescein $(a)$. Color changes after adding $40 \%$ $\mathrm{NaOH}$ into the hollow-(H-) PMO-FDA dispersion: $(b)$ upon addition of $\mathrm{NaOH} ;(c)$ after $1 \mathrm{~h}$; and $(d)$ after tenfold dilution. B Scheme of a solid sandwich immunoassay using H-PMO biolabels [88]

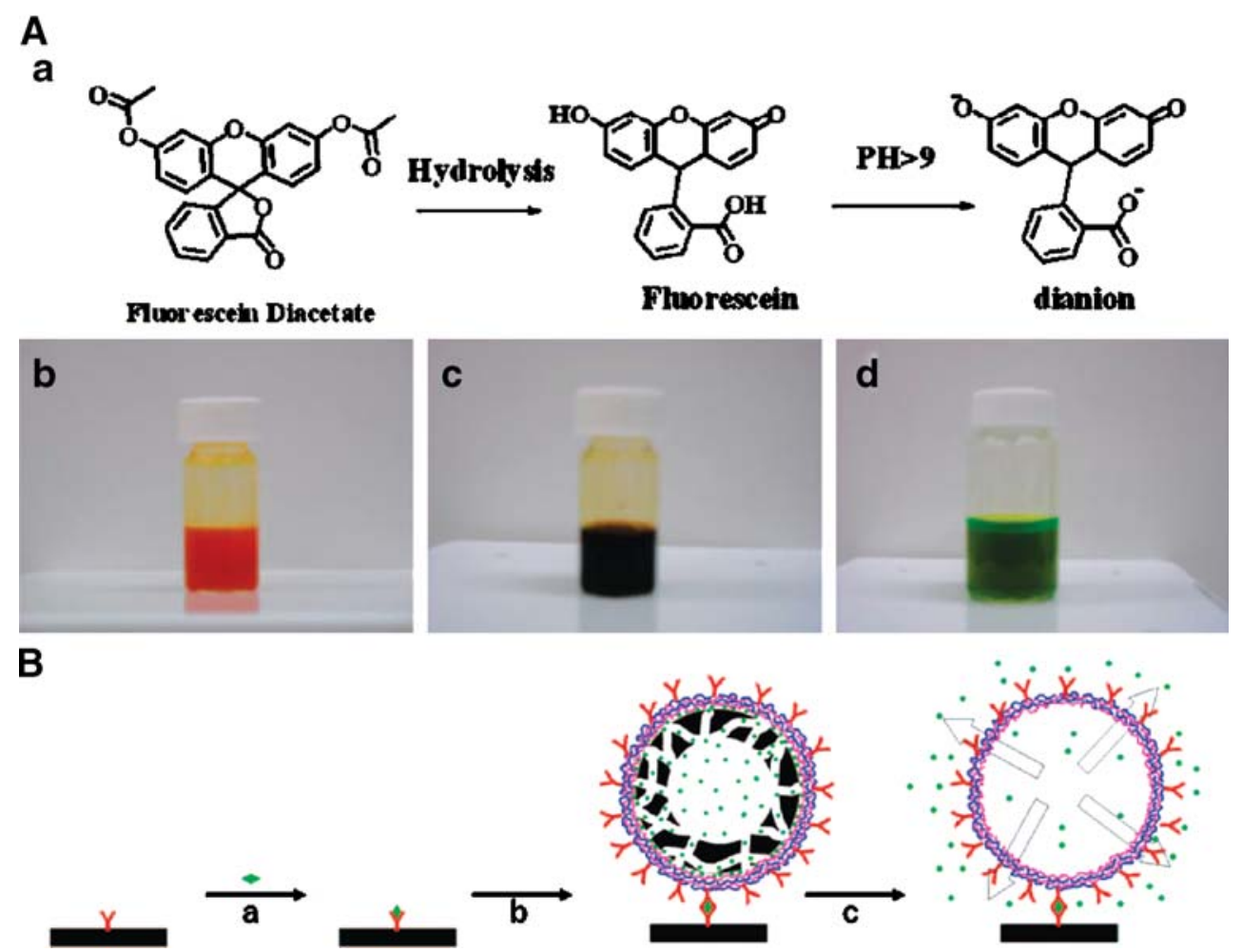


Fluorescence lifetime (FLT) is a robust fluorescence parameter that is not affected by static quenching and variations in fluorophore concentration. It has great potential in multiplexed biosensing because the background fluorescence from the biological sample matrix, which usually has a very short lifetime, can be discriminated easily, and the lifetime is another parameter besides the fluorescence intensity that can be detected to increase the throughput of multiplexing. Lanthanide chelates with submicrosecond to millisecond lifetimes are common labeling reagents for this type of measurement, but their lifetimes are so long that they have very limited photon turnover rates and their fluorescence is also weaker than organic dyes, resulting in a low detection sensitivity [91, 92]. To increase the signal intensity, silica nanoparticles were synthesized that contained thousands of fluorescent lanthanide chelates for sensitive detection of the prostatespecific antigen using time-resolved fluorescence bioassay in a 96-well plate [91, 92]. A detection limit of $7.0 \mathrm{pg} / \mathrm{mL}$ was achieved [92]. Nanocrystals are good labels for FLT measurement due to their relatively long lifetime and high photon turnover rate. It has been demonstrated that QDs can act as the energy acceptors for lanthanide ions, resulting in a thousandfold increase in the QD luminescence decay time. These lanthanide-QD systems were shown to be highly sensitive tools for time-resolved fluoro-immunoassays [93].

Nanocrystals are also suitable candidates for multiphoton fluorescence measurement [94, 95]. In this type of nonlinear optical technique, multiple photons with low energies are absorbed simultaneously to produce photon emission at shorter wavelengths. Multiphoton fluorescence requires a tightly focused incident beam to generate enough intensity for up-converted emission, and thus allows highly localized fluorescence detection. It also offers advantages such as irradiation in the far-red and near-infrared spectral regions, which lowers background absorption and scattering, and little photobleaching. Detection and differentiation of tumor vessels in mice were successfully performed with multiphoton microscopy using QD470, QD590, and QD660 [95]. Water-soluble CdSe/ZnS QDs were synthesized that had two-photon action cross-sections as high as 47,000 Goeppert-Mayer units - claimed to be the largest of any label used in multiphoton microscopy-and they enabled the detection of target analytes at greater depths (down to hundreds of micrometers) inside tissues or animals than possible with traditional organic dyes [95].

\section{Conclusion}

Even though nanomaterials are promising labeling agents in biosensing, they still require improvements in order to obtain robust and practical applications. For example, the chemical synthesis of inorganic nanomaterials in solution often yields a large distribution of particle sizes and shapes, which may lead to nanomaterials with heterogeneous optical properties. In addition, water solubility, surface functionalization, and chemical purity can all affect the performance and stability of nanomaterials in biosensing. To obtain better nanomaterials for biosensing, other than continuously improving the synthetic and modification strategies, analytical characterization and purification of nanomaterials can play significant roles.

Various analytical separation technologies can be applied to resolve or characterize nanomaterials with different sizes, shapes, or even surface modifications. Successful separations of gold/silver nanoparticles or QDs based on their sizes or surface charges have been achieved using slab-gel electrophoresis, isoelectric focusing, or capillary electrophoresis (CE) [96-102]. While the slab-gel technique provides the ability to collect the purified nanoproducts, CE offers fast separation speed and low sample consumption, allowing in situ monitoring of the synthesis process for better product quality control. Other separation technologies - for instance recycling size-exclusion chromatography, sedimentation field flow fractionation (FFF), and diafiltration-have been shown to be effective for the size-based separation of nanoparticles and the removal of impurities during nanomaterial preparation [103-105].

In conclusion, nanomaterials possess great potential in relation to biosensing, offering improved sensitivity and assay simplicity. Their performances can be enhanced by improving their purity and narrowing their size/shape/ conjugation distributions, which can be achieved with various separation techniques. Thus, nanomaterials could provide a great contribution to the development of analytical science, and also benefit to a large extent from modern analytical chemistry.

Open Access This article is distributed under the terms of the Creative Commons Attribution Noncommercial License which permits any noncommercial use, distribution, and reproduction in any medium, provided the original author(s) and source are credited.

\section{References}

1. Ruan G, Agrawal A, Smith AM et al. (2006) Rev Fluoresc 3:181-193

2. Gao X, Yang L, Petros JA et al. (2005) Curr Opin Biotechnol 16:63-72

3. Smith AM, Nie S (2004) Analyst 129:672-677

4. Biju V, Itoh T, Anas A et al. (2008) Anal Bioanal Chem 391:2469-2495

5. Klostranec JM, Chan WCW (2006) Adv Mater 18:1953-1964 
6. Bakalova R, Zhelev Z, Ohba H et al. (2005) J Am Chem Soc 127:9328-9329

7. Ornberg RL, Harper TF, Liu H (2005) Nat Methods 2:79-81

8. Genin E, Carion O, Mahler B et al. (2008) J Am Chem Soc 130:8596-8597

9. Soman CP, Giorgio TD (2008) Langmuir 24:4399-4404

10. Chan P, Yuen T, Ruf F et al. (2005) Nucleic Acids Res 33:e161/ $1-\mathrm{e} 161 / 8$

11. Edgar R, McKinstry M, Hwang J et al. (2006) Proc Natl Acad Sci USA 103:4841-4845

12. Nichkova M, Dosev D, Davies AE et al. (2007) Anal Lett 40:1423-1433

13. Karlin-Neumann G, Sedova M, Falkowski M et al. (2007) Met Mol Biol 374:239-251

14. Shepard JRE (2006) Anal Chem 78:2478-2486

15. Ho Y-P, Kung MC, Yang S et al. (2005) Nano Lett 5:16931697

16. Bentzen EL, Tomlinson ID, Mason J et al. (2005) Bioconjug Chem 16:1488-1494

17. Travert-Branger N, Dubois F, Carion O et al. (2008) Langmuir 24:3016-3019

18. Susumu K, Uyeda HT, Medintz IL et al. (2007) J Am Chem Soc 129:13987-13996

19. Kairdolf BA, Mancini MC, Smith AM et al. (2008) Anal Chem 80:3029-3034

20. Smith JE, Wang L, Tan W (2006) Trends Anal Chem 25:848855

21. Yao G, Wang L, Wu Y et al. (2006) Anal Bioanal Chem 385:518-524

22. Mechery SJ, Zhao XJ, Wang L et al. (2006) Chem Asian J 1:384-390

23. Wang L, Yang C, Tan W (2005) Nano Lett 5:37-43

24. Zhao X, Tapec-Dytioco R, Tan W (2003) J Am Chem Soc 125:11474-11475

25. Qhobosheane M, Santra S, Zhang P et al. (2001) Analyst 126:1274-1278

26. Bagwe RP, Hilliard LR, Tan W (2006) Langmuir 22:4357-4362

27. Sathe TR, Agrawal A, Nie S (2006) Anal Chem 78:5627-5632

28. Law W-C, Yong K-T, Roy I et al. (2008) J Phys Chem C 112:7972-7977

29. Insin N, Tracy JB, Lee H et al. (2008) ACS Nano 2:197-202

30. Selvan ST, Patra PK, Ang CY et al. (2007) Angew Chem Int Ed 46:2448-2452

31. Mangolini L, Kortshagen U (2007) Adv Mater 19:2513-2519

32. Jurbergs D, Rogojina E, Mangolini L et al. (2006) Appl Phys Lett 88:233116/1-233116/3

33. Fujioka K, Hiruoka M, Sato K et al. (2008) Nanotechnology 19:415102/1-415102/7

34. Li QS, Zhang RQ, Lee ST et al. (2008) J Chem Phys 128:244714/1-244714/5

35. Erogbogbo F, Yong K-T, Roy I et al. (2008) ACS Nano 2:873878

36. Li QS, Zhang RQ, Lee ST et al. (2008) Appl Phys Lett 92:053107/1-053107/3

37. Aslan K, Gryczynski I, Malicka J et al. (2005) Curr Opin Biotechnol 16:55-62

38. Geddes Chris D, Gryczynski I, Malicka J et al. (2003) Comb Chem High Throughput Screen 6:109-117

39. DeBono RF, Helluy A, Heimlich M et al. (1993) Sens Actuators B 11:487-497

40. Matveeva EG, Gryczynski Z, Lakowicz JR (2005) J Immunol Methods 302:26-35

41. Matveeva E, Gryczynski Z, Malicka J et al. (2004) Anal Biochem 334:303-311

42. Geddes CD, Cao H, Gryczynski I et al. (2003) J Phys Chem A 107:3443-3449
43. Lakowicz JR (2001) Anal Biochem 298:1-24

44. Johnson E, Aroca R (1991) Can J Chem 69:1728-1731

45. Zhang J, Matveeva E, Gryczynski I et al. (2005) J Phys Chem B 109:7969-7975

46. Aslan K, Lakowicz Joseph R, Geddes Chris D (2005) Anal Bioanal Chem 382:926-933

47. Pompa PP, Martiradonna L, Della Torre A et al. (2006) Nat Nanotechnol 1:126-130

48. Ray K, Chowdhury Mustafa H, Lakowicz Joseph R (2007) Anal Chem 79:6480-6487

49. Zhang Y, Aslan K, Previte MJR et al. (2007) Appl Phys Lett 90:173116/1-173116/3

50. Aslan K, Huang J, Wilson Gerald M et al. (2006) J Am Chem Soc 128:4206-4207

51. Lakowicz Joseph R, Shen Y, D'Auria S et al. (2002) Anal Biochem 301:261-277

52. Sabanayagam CR, Lakowicz JR (2007) Nucleic Acids Res 35 e13/1-e13/9

53. Aslan K, Huang J, Wilson Gerald M et al. (2006) J Am Chem Soc 128:4206-4207

54. Tsai SP, Wong A, Mai E et al. (2003) Nucleic Acids Res 31:e25/ $1-\mathrm{e} 25 / 7$

55. Baugh LR, Hill AA, Brown EL et al. (2001) Nucleic Acids Res 29:e29/1-e29/9

56. Zhang J, Fu Y, Liang D et al. (2008) Langmuir 24:1245212457

57. Aslan K, Holley P, Geddes Chris D (2006) J Immunol Methods 312:137-147

58. Aslan K, Geddes CD (2005) Anal Chem 77:8057-8067

59. Aslan K, Previte Michael JR, Zhang Y et al. (2008) Anal Chem 80:4125-4132

60. Ellerbrok H, Nattermann H, Ozel $M$ et al. (2002) FEMS Microbiol Lett 214:51-59

61. Asian K, Lakowicz Joseph R, Szmacinski H et al. (2004) J Fluoresc 14:677-679

62. Aslan K, Wu M, Lakowicz Joseph R et al. (2007) J Am Chem Soc 129:1524-1525

63. Sapsford KE, Berti L, Medintz IL (2006) Angew Chem Int Ed 45:4562-4588

64. Dubertret B, Calame M, Libchaber AJ (2001) Nat Biotechnol 19:365-370

65. Maxwell DJ, Taylor JR, Nie S (2002) J Am Chem Soc 124:9606-9612

66. Zhang N, Liu Y, Tong L et al. (2008) Analyst 133:1176-1181

67. Tang B, Zhang N, Chen Z et al. (2008) Chem Eur J 14:522528

68. Zhang J, Fu Y, Lakowicz JR (2007) J Phys Chem C 111:5056

69. Zhang J, Fu Y, Chowdhury MH et al. (2007) J Phys Chem C 111:11784-11792

70. Curutchet C, Franceschetti A, Zunger A et al. (2008) J Phys Chem C 112:13336-13341

71. Algar WR, Krull UJ (2008) Anal Bioanal Chem 391:1609-1618

72. Suzuki M, Husimi Y, Komatsu H et al. (2008) J Am Chem Soc 130:5720-5725

73. Chen HH, Ho Y-P, Jiang X et al. (2008) Mol Therapy 16:324 332

74. Zhang C-Y, Johnson LW (2007) Anal Chem 79:7775-7781

75. Komarala VK, Bradley AL, Rakovich YP et al. (2007) Proc SPIE 6641:66410Y/1-66410Y/8

76. Tang B, Cao L, Xu K et al. (2008) Chem Eur J 14:3637-3644

77. Gao D, Wang Z, Liu B et al. (2008) Anal Chem (in press)

78. Wang Y, Liu B (2007) Anal Chem 79:7214-7220

79. Wang L, Tan W (2006) Nano Lett 6:84-88

80. Wang L, Zhao W, O’Donoghue MB et al. (2007) Bioconjug Chem 18:297-301 
81. Cui D, Pan B, Zhang H et al. (2008) Anal Chem 80:7996-8001

82. Yang R, Tang Z, Yan J et al. (2008) Anal Chem 80:7408-7413

83. Pan B, Cui D, Ozkan CS et al. (2008) J Phys Chem C 112:939944

84. Hedderman TG, Keogh SM, Chambers G et al. (2004) J Phys Chem B 108:18860-18865

85. Liu Z, Sun X, Nakayama-Ratchford N et al. (2007) ACS Nano $1: 50-56$

86. Nakayama-Ratchford N, Bangsaruntip S, Sun X et al. (2007) J Am Chem Soc 129:2448-2449

87. Wu C, Zheng Y, Szymanski C et al. (2008) J Phys Chem C 112:1772-1781

88. Cai W, Gentle IR, Lu GQ et al. (2008) Anal Chem 80:54015406

89. Li J, Zhang T, Ge J et al. (2008) Angew Chem Int Ed (accepted)

90. Son DH, Hughes SM, Yin Y et al. (2004) Science 306:10091012

91. Zhang H, Xu Y, Yang W et al. (2007) Chem Mater 19:58755881

92. Ye Z, Tan M, Wang G et al. (2004) Anal Chem 76:513-518

93. Charbonniere LJ, Hildebrandt N, Ziessel RF et al. (2006) J Am Chem Soc 128:12800-12809
94. Stroh M, Zimmer JP, Duda DG et al. (2005) Nat Med 11:678682

95. Larson DR, Zipfel WR, Williams RM et al. (2003) Science 300:1434-1437

96. Xu X, Caswell KK, Tucker E et al. (2007) J Chromatogr A 1167:35-41

97. Hanauer M, Pierrat S, Zins I et al. (2007) Nano Lett 7:28812885

98. Sperling RA, Pellegrino T, Li JK et al. (2006) Adv Func Mater 16:943-948

99. Liu F-K (2007) J Chromatogr A 1167:231-235

100. Pereira M, Lai EPC, Hollebone B (2006) J Chromatogr A 1133:340-346

101. Song X, Li L, Qian H et al. (2006) Electrophoresis 27:1341-1346

102. Arnaud I, Abid J-P, Roussel C et al. (2005) Chem Comm 787788

103. Kim ST, Kang DY, Lee S et al. (2007) J Liq Chromatogr R T 30:2533-2544

104. Sweeney SF, Woehrle GH, Hutchison JE (2006) J Am Chem Soc 128:3190-3197

105. Al-Somali AM, Krueger KM, Falkner JC et al. (2004) Anal Chem 76:5903-5910 